\title{
Hand Tremors Reduction Based on Integral Intelligent Filter Computed Torque Controller
}

\author{
${ }^{1}$ Mahdi Mirshekaran, ${ }^{1}$ Farzin Piltan, ${ }^{1,2}$ Nasri Sulaiman, ${ }^{1}$ Alireza Salehi, ${ }^{1}$ Meysam \\ Kazeminasab and ${ }^{1}$ Zahra Esmaeili \\ ${ }^{1}$ Intelligent System and Robotic Lab, Iranian Institute of Advance Science and \\ Technology (IRAN SSP), Shiraz/Iran \\ ${ }^{2}$ Department of Electrical and Electronic Engineering, Faculty of Engineering, \\ University Putra Malaysia, Malaysia \\ Email: piltan_f@iranssp.com,WWW.IRANSSP.COM/english
}

\begin{abstract}
Computed torque controller (CTC) is one of the types of feedback linearization nonlinear controller. This controller works very well in certain positions. However this controller has many advantages in certain conditions but it has challenges in uncertainty. The main challenge in CTC is fluctuations in uncertainties. In this research low pass filter is used to reduce the fluctuations in CTC. To improve the result of this controller intelligent CTC is recommended based on fuzzy logic engineering. In this research fuzzy logic theory is used to tune the new low pass filter CTC coefficients. The process of setting of integral intelligent Computed Torque Controller can be determined as an optimization task. Over the years, use of intelligent strategies for tuning of these controllers has been growing. This research is used to reduce or eliminate the computed torque controller problem based on low pass filter and fuzzy logic theory to control of flexible robot manipulator system and testing of the quality of process control in the simulation environment of MATLAB/SIMULINK Simulator.
\end{abstract}

Keywords: Integral filter, artificial intelligence, fuzzy logic control, computed torque control, robot manipulator, hand tremors

\section{Introduction}

Continuum robots represent a class of robots that have a biologically inspired form characterized by flexible backbones and high degrees-of-freedom structures [1-3]. Theoretically, the compliant nature of a continuum robot provides infinite degrees of freedom to these devices. However, there is a limitation set by the practical inability to incorporate infinite actuators in the device. Most of these robots are consequently under actuated (in terms of numbers of independent actuators) with respect to their anticipated tasks. In other words they must achieve a wide range of configurations with relatively few control inputs. This is partly due to the desire to keep the body structures (which, unlike in conventional rigid-link manipulators or fingers, are required to directly contact the environment) "clean and soft", but also to exploit the extra control authority available due to the continuum contact conditions with a minimum number of actuators. For example, the Octarm VI continuum manipulator, discussed frequently in this paper, has nine independent actuated degrees-of-freedom with only three sections. Continuum manipulators differ fundamentally from rigid-link and hyper-redundant robots by having an unconventional structure that lacks links and joints. Hence, standard techniques like the Denavit-Hartenberg (D-H) algorithm cannot be directly applied for developing continuum arm kinematics. Moreover, the design of 
each continuum arm varies with respect to the flexible backbone present in the system, the positioning, type and number of actuators. The constraints imposed by these factors make the set of reachable configurations and nature of movements unique to every continuum robot. This makes it difficult to formulate generalized kinematic or dynamic models for continuum robot hardware. Thus, the kinematics (i.e. geometry based modeling) of a quite general set of prototypes of continuum manipulators has been developed and basic control strategies now exist based on these. The development of analytical models to analyze continuum arm dynamics (i.e. physics based models involving forces in addition to geometry) is an active, ongoing research topic in this field. From a practical perspective, the modeling approaches currently available in the literature prove to be very complicated and a dynamic model which could be conveniently implemented in an actual device's real-time controller has not been developed yet. The absence of a computationally tractable dynamic model for these robots also prevents the study of interaction of external forces and the impact of collisions on these continuum structures. This impedes the study and ultimate usage of continuum robots in various practical applications like grasping and manipulation, where impulsive dynamics [410] are important factors. Although continuum robotics is an interesting subclass of robotics with promising applications for the future, from the current state of the literature, this field is still in its stages of inception.

Controller (control system) is a device which can sense information from linear or nonlinear system (e.g., robot arm) to improve the systems performance and the immune system behavior [11-15]. In feedback control system considering that there are many disturbances and also variable dynamic parameters something that is really necessary is keeping plant variables close to the desired value. Feedback control system development is the most important thing in many different fields of safety engineering. The main targets in design control systems are safety stability, good disturbance rejection to reach the best safety, and small tracking error[14-15]. At present, in some applications robot arms are used in unknown and unstructured environment, therefore strong mathematical tools used in new control methodologies to design nonlinear robust controller with an acceptable safety performance (e.g., minimum error, good trajectory, disturbance rejection). According to the control theory, systems' controls are divided into two main groups: conventional control theory and soft computing control theory. Conventional control theories are work based on manipulator dynamic model. This technique is highly sensitive to the knowledge of all parameters of nonlinear robot manipulator's dynamic equation. Conventional control theory is divided into two main groups: linear control theory and nonlinear control theory. Soft computing (intelligent) control theory is free of some challenges associated to conventional control theory. This technique is worked based on intelligent control theory. This theory is divided into the following groups: fuzzy logic theory, neural network theory, genetic algorithm and neuro-fuzzy theory.

One of the most important nonlinear safety controllers is computed torque methodology which is used in nonlinear certain systems. This methodology is used in wide range areas such as in safety control access process; in aerospace applications and in robotics because this methodology can solve some main challenging topics in safety control access such as resistivity to the external disturbance and stability. Even though, this methodology is used in wide range areas but, pure computed torque method has an important drawbacks beside uncertain system and also in presence of external disturbance. Uncertainty in system can causes some problems about safety in industrial factory [15]. Although the fuzzy-logic control is not a new technique, its application in this current research is considered to be novel since it aimed for an automated dynamic-less response rather than for the traditional objective of uncertainties compensation[11-15]. The intelligent tracking control using the 
fuzzy-logic technique provides a cost-and-time efficient control implementation due to the automated dynamic-less input. This in turn would further inspire multi-uncertainties testing for continuum robot [13-15].

A low-pass filter is a filter that passes low-frequency signals and attenuates (reduces the amplitude of) signals with frequencies higher than the cutoff frequency. The actual amount of attenuation for each frequency varies depending on specific filter design. It is sometimes called a high-cut filter, or treble cut filter in audio applications. A low-pass filter is the opposite of a high-pass filter. A band-pass filter is a combination of a low-pass and a high-pass.

Low-pass filters exist in many different forms, including electronic circuits (such as a hiss filter used in audio), anti-aliasing filters for conditioning signals prior to analog-to-digital conversion, digital filters for smoothing sets of data, acoustic barriers, blurring of images, and so on. The moving average operation used in fields such as finance is a particular kind of low-pass filter, and can be analyzed with the same signal processing techniques as are used for other low-pass filters. Low-pass filters provide a smoother form of a signal, removing the short-term fluctuations, and leaving the longer-term trend. An optical filter can correctly be called a low-pass filter, but conventionally is called a longpass filter (low frequency is long wavelength), to avoid confusion.

In this research the active low pass filter is used to eliminate the high frequency oscillation in conventional computed torque controller in presence of uncertainty and fuzzy logic controller is used to tune modified computed torque coefficients.

This paper is organized as follows; section 2, is served as an introduction to the dynamic of continuum robot. Part 3 introduces and describes the methodology algorithm. Section 4 presents the simulation results and discussion of this algorithm applied to a continuum robot and the last part is described as conclusion.

\section{Theory}

Dynamic Formulation of Continuum Robot: The Continuum section analytical model developed here consists of three modules stacked together in series. In general, the model will be a more precise replication of the behavior of a continuum arm with a greater of modules included in series. However, we will show that three modules effectively represent the dynamic behavior of the hardware, so more complex models are not motivated. Thus, the constant curvature bend exhibited by the section is incorporated inherently within the model. The model resulting from the application of Lagrange's equations of motion obtained for this system can be represented in the form

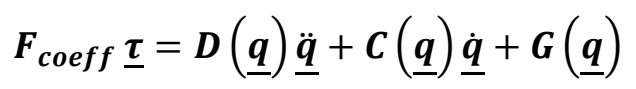

where $\tau$ is a vector of input forces and $\mathrm{q}$ is a vector of generalized co-ordinates. The force coefficient matrix $F_{\text {coeff }}$ transforms the input forces to the generalized forces and torques in the system. The inertia matrix, $D$ is composed of four block matrices. The block matrices that correspond to pure linear accelerations and pure angular accelerations in the system (on the top left and on the bottom right) are symmetric. The matrix $C$ contains coefficients of the first order derivatives of the generalized co-ordinates. Since the system is nonlinear, many elements of $C$ contain first order derivatives of the generalized co-ordinates. The remaining terms in the dynamic equations resulting from gravitational potential energies and spring 
energies are collected in the matrix $G$. The coefficient matrices of the dynamic equations are given below,

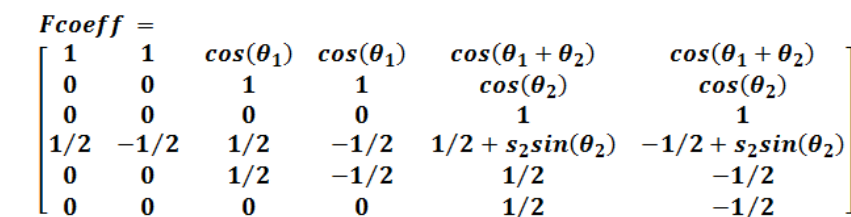

(2)

(3)

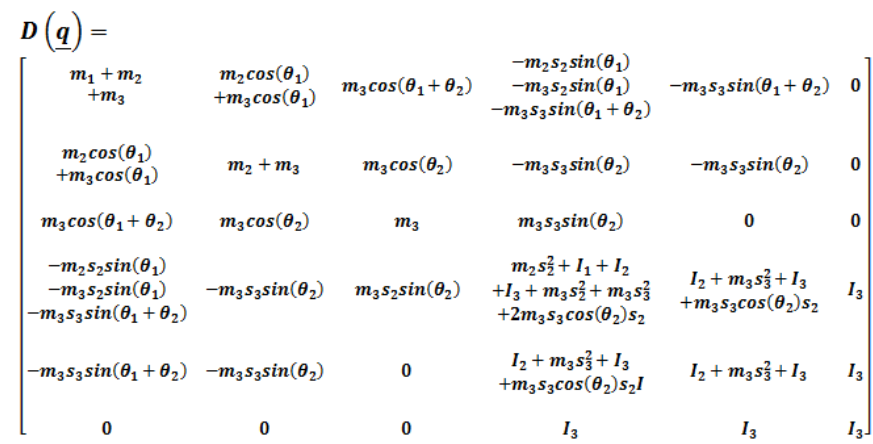

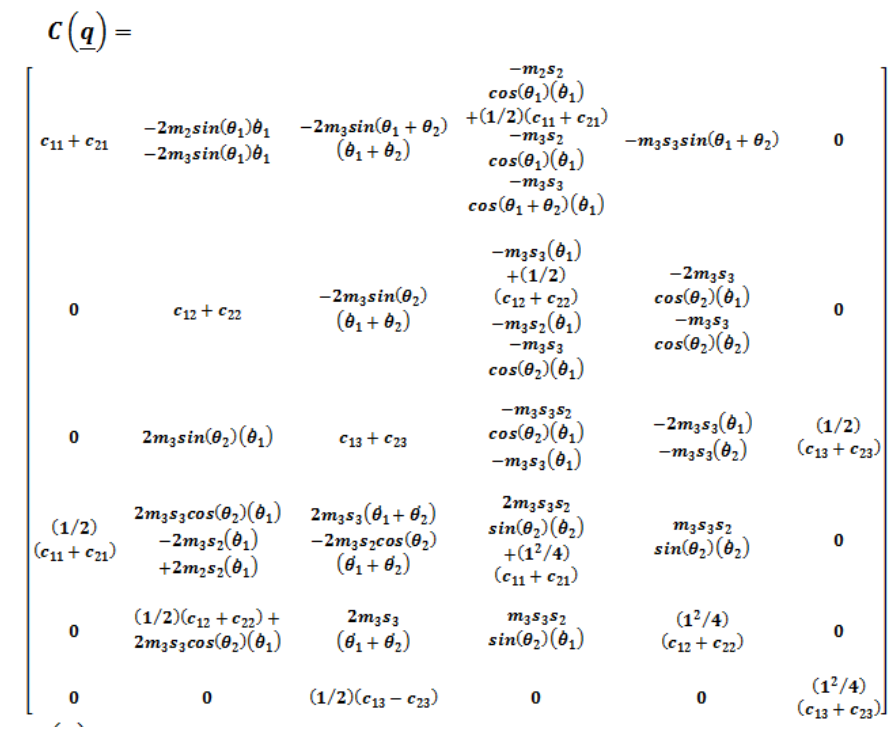

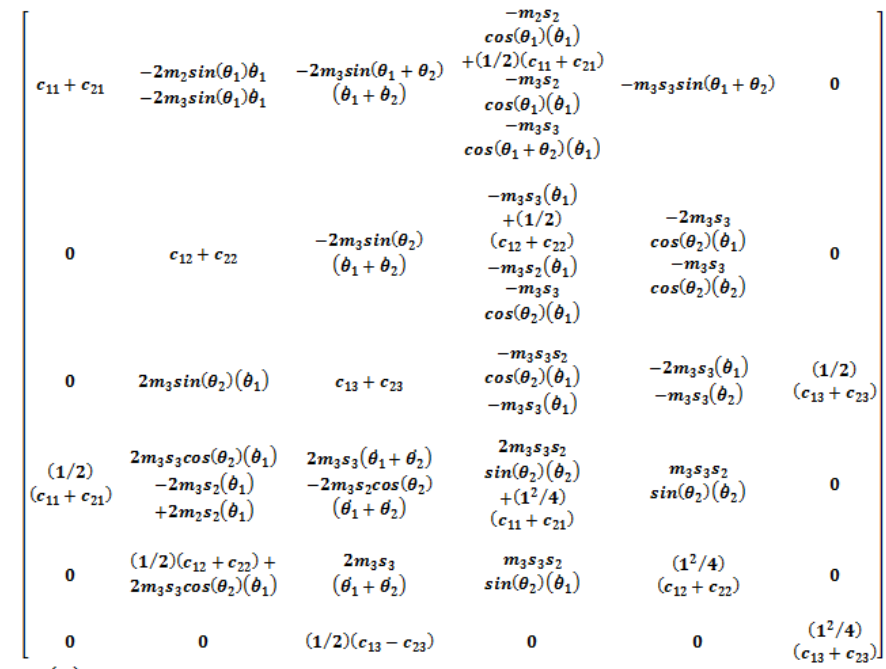

$\boldsymbol{G}(\underline{q})=$

(5)

$$
\left[\begin{array}{c}
-m_{1} g-m_{2} g+k_{11}\left(s_{1}+(1 / 2) \theta_{1}-s_{01}\right)+k_{21}\left(s_{1}-(1 / 2) \theta_{1}-s_{01}\right)-m_{3} g \\
-m_{2} g \cos \left(\theta_{1}\right)+k_{12}\left(s_{2}+(1 / 2) \theta_{2}-s_{02}\right)+k_{22}\left(s_{2}-(1 / 2) \theta_{2}-s_{02}\right)-m_{3} g \cos \left(\theta_{1}\right) \\
-m_{3} g \cos \left(\theta_{1}+\theta_{2}\right)+k_{13}\left(s_{3}+(1 / 2) \theta_{3}-s_{03}\right)+k_{23}\left(s_{3}-(1 / 2) \theta_{3}-s_{03}\right) \\
m_{2} s_{2} g \sin \left(\theta_{1}\right)+m_{3} s_{3} g \sin \left(\theta_{1}+\theta_{2}\right)+m_{3} s_{2} g \sin \left(\theta_{1}\right)+k_{11}\left(s_{1}+(1 / 2) \theta_{1}-s_{01}\right)(1 / 2) \\
+k_{21}\left(s_{1}-(1 / 2) \theta_{1}-s_{01}\right)(-1 / 2) \\
m_{3} s_{3} g \sin \left(\theta_{1}+\theta_{2}\right)+k_{12}\left(s_{2}+(1 / 2) \theta_{2}-s_{02}\right)(1 / 2)+k_{22}\left(s_{2}-(1 / 2) \theta_{2}-s_{02}\right)(-1 / 2) \\
k_{13}\left(s_{3}+(1 / 2) \theta_{3}-s_{03}\right)(1 / 2)+k_{23}\left(s_{3}-(1 / 2) \theta_{3}-s_{03}\right)(-1 / 2)
\end{array}\right]
$$

\section{Methodology}

Computed torque controller (CTC) is a powerful nonlinear controller which it widely used in control of nonlinear systems. It is based on feedback linearization and computes the 
required arm torques using the nonlinear feedback control law. This controller works very well when all dynamic and physical parameters are known but when the robot has variation in dynamic parameters, in this situation the controller has no acceptable trajectory performance. In practice, most of physical systems (e.g., continuum robot) parameters are unknown or time variant, therefore, computed torque like controller used to compensate dynamic equation of continuum robot. When all dynamic and physical parameters are known, computed torque controller works fantastically; practically a large amount of systems have uncertainties, therefore computed torque like controller is the best case to solve this challenge. The central idea of computed torque controller (CTC) is feedback linearization so, originally this algorithm is called feedback linearization controller. It has assumed that the desired motion trajectory for the manipulator $\boldsymbol{q}_{\boldsymbol{d}}(\boldsymbol{t})$, as determined, by a path planner. Defines the tracking error as:

$$
e(t)=q_{d}(t)-q_{a}(t)
$$

Where e(t) is error of the plant, $\boldsymbol{q}_{\boldsymbol{d}}(\boldsymbol{t})$ is desired input variable, that in our system is desired displacement, $\boldsymbol{q}_{\boldsymbol{a}}(\boldsymbol{t})$ is actual displacement. If an alternative linear state-space equation in the form $\dot{\boldsymbol{x}}=\boldsymbol{A} \boldsymbol{x}+\boldsymbol{B} \boldsymbol{U}$ can be defined as

$$
\dot{x}=\left[\begin{array}{ll}
\mathbf{0} & \mathbf{I} \\
\mathbf{0} & \mathbf{0}
\end{array}\right] \boldsymbol{x}+\left[\begin{array}{l}
\mathbf{0} \\
\boldsymbol{I}
\end{array}\right] \boldsymbol{U}
$$

With $\boldsymbol{U}=-\boldsymbol{D}^{-\mathbf{1}}(\boldsymbol{q}) \cdot \boldsymbol{N}(\boldsymbol{q}, \dot{\boldsymbol{q}})+\boldsymbol{D}^{-\mathbf{1}}(\boldsymbol{q}) \cdot \boldsymbol{\tau}$ and this is known as the Brunousky canonical form. By equation (6) and (7) the Brunousky canonical form can be written in terms of the state $x=\left[e^{T} \dot{e}^{T}\right]^{T}$ as:

$$
\frac{d}{d t}\left[\begin{array}{l}
e \\
\dot{e}
\end{array}\right]=\left[\begin{array}{ll}
0 & I \\
0 & 0
\end{array}\right] \cdot\left[\begin{array}{l}
e \\
\dot{e}
\end{array}\right]+\left[\begin{array}{l}
0 \\
I
\end{array}\right] U
$$

With

$$
U=\ddot{q}_{d}+D^{-1}(q) \cdot\{N(q \cdot \dot{q})-\tau\}
$$

Then compute the required arm torques using inverse of equation (9), is;

$$
\tau=D(q)\left(\ddot{q_{d}}-U\right)+N(\dot{q}, q)
$$

This is a nonlinear feedback control law that guarantees tracking of desired trajectory. Selecting proportional-plus-derivative (PD) feedback for $\mathrm{U}(\mathrm{t})$ results in the PD-computed torque controller;

$$
\tau=D(q)\left(\ddot{q}_{d}+K_{v} \dot{e}+K_{p} e\right)+N(q, \dot{q})
$$

and the resulting linear error dynamics are

$$
\left(\ddot{q}_{d}+K_{v} \dot{e}+K_{p} e\right)=0
$$


According to the linear system theory, convergence of the tracking error to zero is guaranteed. Where $\boldsymbol{K}_{\boldsymbol{p}}$ and $\boldsymbol{K}_{\boldsymbol{v}}$ are the controller gains. Figure 1 shows the computed torque controller for $6 \mathrm{DOF}$ continuum robot manipulator.

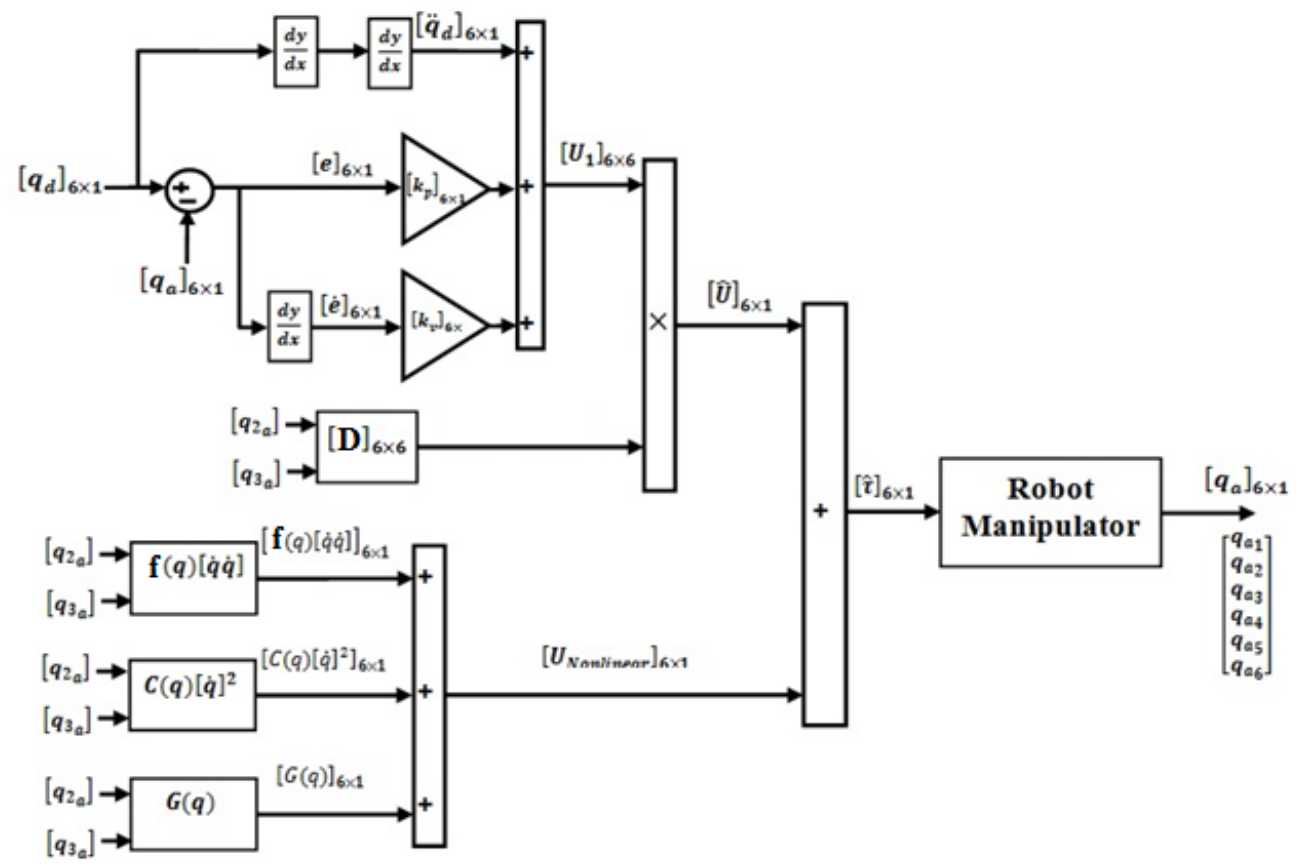

Figure 1. Computed Torque Control: Continuum Robot Manipulator

Based on foundation of fuzzy logic methodology; fuzzy logic controller has played important rule to design nonlinear controller for nonlinear and uncertain systems. However the application area for fuzzy control is really wide, the basic form for all command types of controllers consists of;

Input fuzzification (binary-to-fuzzy [B/F] conversion)

Fuzzy rule base (knowledge base), Inference engine and Output defuzzification (fuzzy-tobinary [F/B] conversion). Figure 2 shows a fuzzy controller block diagram.

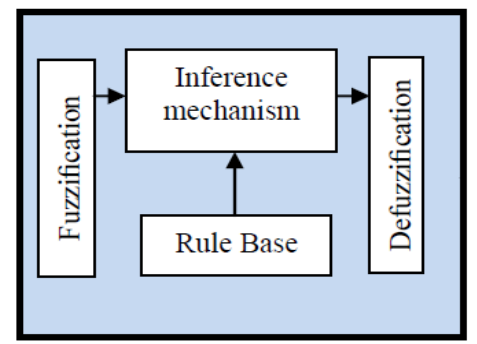

Figure 2. Fuzzy Controller Part

The fuzzy inference engine offers a mechanism for transferring the rule base in fuzzy set which it is divided into two most important methods, namely, Mamdani method and Sugeno method. Mamdani method is one of the common fuzzy inference systems and he designed one of the first fuzzy controllers to control of system engine. Mamdani's fuzzy inference 
system is divided into four major steps: fuzzification, rule evaluation, aggregation of the rule outputs and defuzzification. Michio Sugeno use a singleton as a membership function of the rule consequent part. The following definition shows the Mamdani and Sugeno fuzzy rule base

\section{if $x$ is $A$ and $y$ is $B$ then $z$ is $C$ 'mamdani' if $x$ is $A$ and $y$ is $B$ then $z$ is $f(x, y)$ 'sugeno'}

When $x$ and $y$ have crisp values fuzzification calculates the membership degrees for antecedent part. Rule evaluation focuses on fuzzy operation $(A N D / O R)$ in the antecedent of the fuzzy rules. The aggregation is used to calculate the output fuzzy set and several methodologies can be used in fuzzy logic controller aggregation, namely, Max-Min aggregation, Sum-Min aggregation, Max-bounded product, Max-drastic product, Maxbounded sum, Max-algebraic sum and Min-max. Two most common methods that used in fuzzy logic controllers are Max-min aggregation and Sum-min aggregation. Max-min aggregation defined as below;

$$
\mu_{U}\left(x_{k}, y_{k}, U\right)=\mu_{\cup i=1}^{r} F R^{i}\left(x_{k}, y_{k}, U\right)=\max \left\{\min _{i=1}^{r}\left[\mu_{R p q}\left(x_{k}, y_{k}\right), \mu_{p_{m}}(U)\right]\right\}
$$

The Sum-min aggregation defined as below

$$
\mu_{U}\left(x_{k}, y_{k}, U\right)=\mu_{\cup_{i=1}^{r} F R^{i}}\left(x_{k}, y_{k}, U\right)=\sum \min _{i=1}^{r}\left[\mu_{R} p q\left(x_{k}, y_{k}\right), \mu_{p_{m}}(U)\right]
$$

where $r$ is the number of fuzzy rules activated by $x_{k}$ and $y_{k}$ and also $\mu_{\cup_{i=1}^{r} F R^{i}}\left(x_{k}, y_{k}, U\right)$ is a fuzzy interpretation of $i-t h$ rule. Defuzzification is the last step in the fuzzy inference system which it is used to transform fuzzy set to crisp set. Consequently defuzzification's input is the aggregate output and the defuzzification's output is a crisp number. Centre of gravity method (COG) and Centre of area method (COA) are two most common defuzzification methods, which $C O G$ method used the following equation to calculate the defuzzification

$$
\operatorname{Cog}\left(x_{k}, y_{k}\right)=\frac{\sum_{i} U_{i} \sum_{j=1}^{r} \cdot \mu_{u}\left(x_{k}, y_{k}, U_{i}\right)}{\sum_{i} \sum_{j=1}^{r} \cdot \mu_{u}\left(x_{k}, y_{k}, U_{i}\right)}
$$

and $C O A$ method used the following equation to calculate the defuzzification

$$
\operatorname{COA}\left(x_{k}, y_{k}\right)=\frac{\sum_{i} U_{i} \cdot \mu_{u}\left(x_{k}, y_{k}, U_{i}\right)}{\sum_{i} \mu_{U}\left(x_{k}, y_{k}, U_{i}\right)}
$$

Where $\operatorname{COG}\left(x_{k}, y_{k}\right)$ and $\operatorname{COA}\left(x_{k}, y_{k}\right)$ illustrates the crisp value of defuzzification output, $U_{i} \in U$ is discrete element of an output of the fuzzy set, $\mu_{U} \cdot\left(x_{k}, y_{k}, U_{i}\right)$ is the fuzzy set membership function, and $r$ is the number of fuzzy rules.

The main disadvantage of Passive Filters is that the amplitude of the output signal is less than that of the input signal, $i$. e., the gain is never greater than unity and that the load impedance affects the filters characteristics. With passive filter circuits containing multiple stages, this loss in signal amplitude called "Attenuation" can become quiet severe. One way of restoring or controlling this loss of signal is by using amplification through the use 
of Active Filters. As their name implies, Active Filters contain active components such as operational amplifiers, transistors or FET's within their circuit design. They draw their power from an external power source and use it to boost or amplify the output signal.

Filter amplification can also be used to either shape or alter the frequency response of the filter circuit by producing a more selective output response, making the output bandwidth of the filter narrower or even wider. Then the main difference between a "passive filter" and an "active filter" is amplification.

An active filter generally uses an operational amplifier (op-amp) within its design and an Op-amp has high input impedance, low output impedance and a voltage gain determined by the resistor network within its feedback loop. Unlike a passive high pass filter which has in theory an infinite high frequency response, the maximum frequency response of an active filter is limited to the Gain/Bandwidth product (or open loop gain) of the operational amplifier being used. Still, active filters are generally easier to design than passive filters; they produce good performance characteristics, very good accuracy with a steep roll-off and low noise when used with a good circuit design. The most common and easily understood active filter is the Active Low Pass Filter. Its principle of operation and frequency response is exactly the same as those for the previously seen passive filter, the only difference this time is that it uses an op-amp for amplification and gain control. The simplest form of a low pass active filter is to connect an inverting or non-inverting amplifier, the same as those discussed in the Op-amp tutorial, to the basic RC low pass filter circuit as shown in Figure 3.

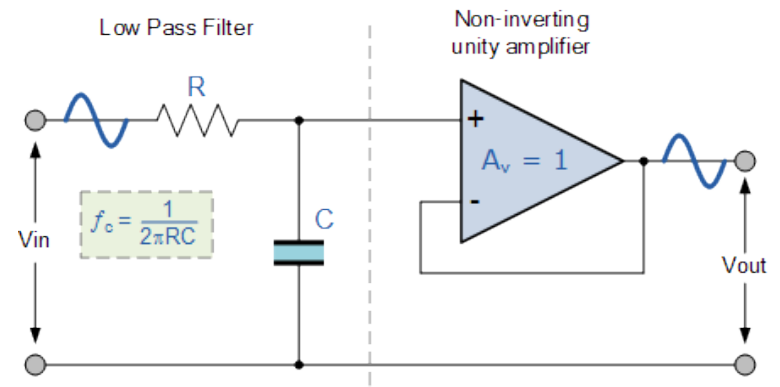

Figure 3. Ideal Low Pass Filter

This first-order low pass active filter, consists simply of a passive RC filter stage providing a low frequency path to the input of a non-inverting operational amplifier. The amplifier is configured as a voltage-follower (Buffer) giving it a DC gain of one, $\mathrm{Av}=+1$ or unity gain as opposed to the previous passive RC filter which has a DC gain of less than unity.

The advantage of this configuration is that the op-amps high input impedance prevents excessive loading on the filters output while its low output impedance prevents the filters cutoff frequency point from being affected by changes in the impedance of the load.

While this configuration provides good stability to the filter, its main disadvantage is that it has no voltage gain above one. However, although the voltage gain is unity the power gain is very high as its output impedance is much lower than its input impedance. If a voltage gain greater than one is required we can use the following filter circuit. As with the passive filter, a first-order Low Pass Active Filter can be converted into a second-order low pass filter simply by using an additional RC network in the input path. The frequency response of the secondorder low pass filter is identical to that of the first-order type except that the stop band roll-off will be twice the first-order filters at $40 \mathrm{~dB} / \mathrm{decade}$ ( $12 \mathrm{~dB} /$ octave). Therefore, the design steps 
required of the second-order active low pass filter are the same. Figure 4 shows the second order nonlinear filter.

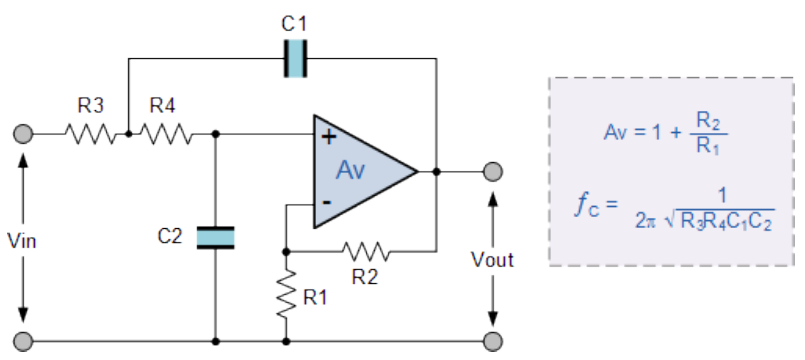

Figure 4. Second Order Nonlinear Filter

To reduce the oscillation in presence of uncertainty the second order active filter is used in conventional computed torque controller. Figure 5 shows the LPF computed torque controller.

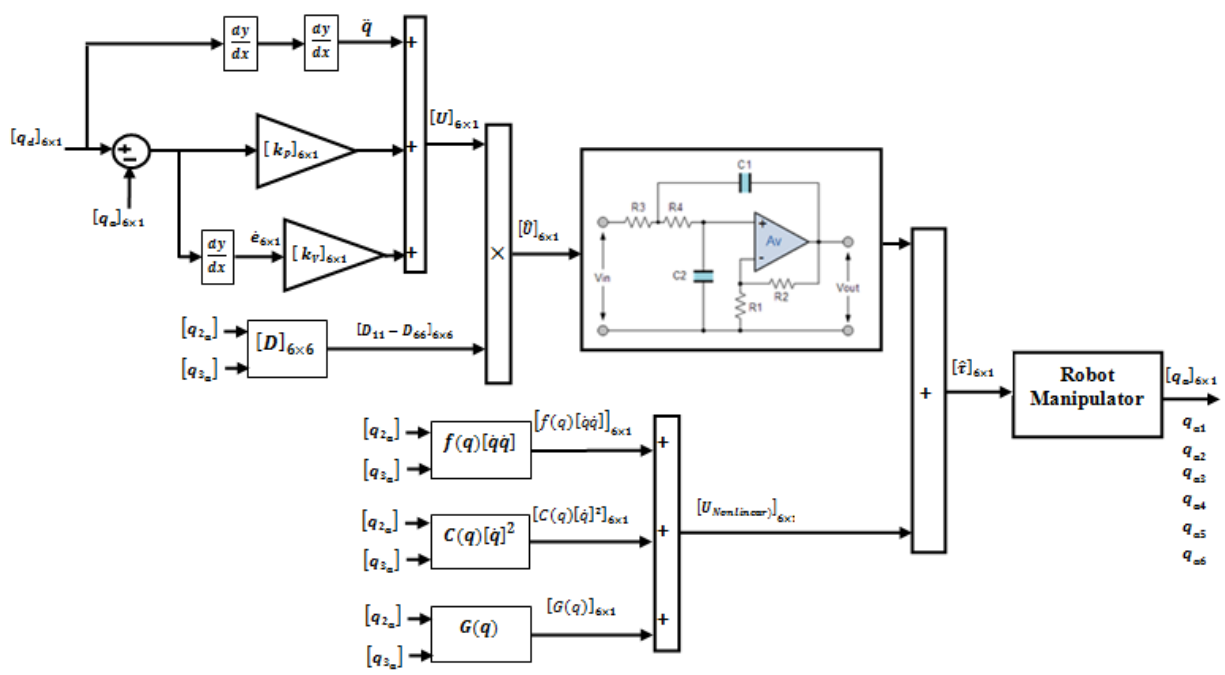

Figure 5. LPF Computed Torque Controller

The standard form of a second-order, low-pass filter is given as:

$$
L P F=\frac{T_{L P(0)} \times\left(\omega_{0}\right)^{2}}{S^{2}+\frac{\omega_{0}}{Q} S+\left(\omega_{0}\right)^{2}}
$$

where $\boldsymbol{T}_{\boldsymbol{L P}(\mathbf{0})}$ is the value of $\boldsymbol{T}_{\boldsymbol{L P}(\boldsymbol{s})}$ at dc, $\boldsymbol{\omega}_{\mathbf{0}}$ is the pole frequency, and $Q$ is the pole $Q$ or the pole quality factor.

Conversely pure computed torque controller is a high-quality nonlinear controller; it has an important problem; nonlinear dynamic formulation in uncertain dynamic parameter. Computed torque controller is a nonlinear controller but it has a challenge in stability and robustness especially in presence of uncertainty and disturbance. Based on literature CTC formulation is written by; 


$$
\tau=D(q)\left(\ddot{q}_{d}+K_{v} \dot{e}+K_{p} e\right)+N(q, \dot{q})
$$

The main challenge in this research is the role of nonlinearity term in presence of uncertainty. To solve this main challenge artificial intelligence based controller is introduce. This type of controller is intelligent therefore design a dynamic of system based on experience knowledge is done by this method. One of the main artificial intelligence techniques is fuzzy logic theory. In this theory the behavior and dynamic of controller is defined by rule base. However defined and number of rule base play important role to design high quality controller but system has limitation to the number of rule base to implementation and the speed of response. This type of controller is used to tuning the LPF computed torque controller's coefficient.

$$
\begin{aligned}
& \tau=D(q)\left(\ddot{q}_{d}+K_{v} \dot{e}+K_{p} e\right)+N(q, \dot{q}) \\
& W=\left(K_{v} \dot{e}+K_{p} e\right) \\
& \tau=D(q)\left(\ddot{q}_{d}+K_{v} \dot{e}+K_{p} e\right)+N(q, \dot{q}) \\
& W_{n e w}=\left((1-\alpha) \times K_{v} \dot{e}+\alpha \times K_{p} e\right) \\
& W_{n e w}=\left(\alpha \times K_{p} e-\alpha \times K_{v} \dot{e}+K_{v} \dot{e}\right)
\end{aligned}
$$

Where $\boldsymbol{\alpha}$ is fuzzy output.

Figure 6 shows the block diagram of proposed method.

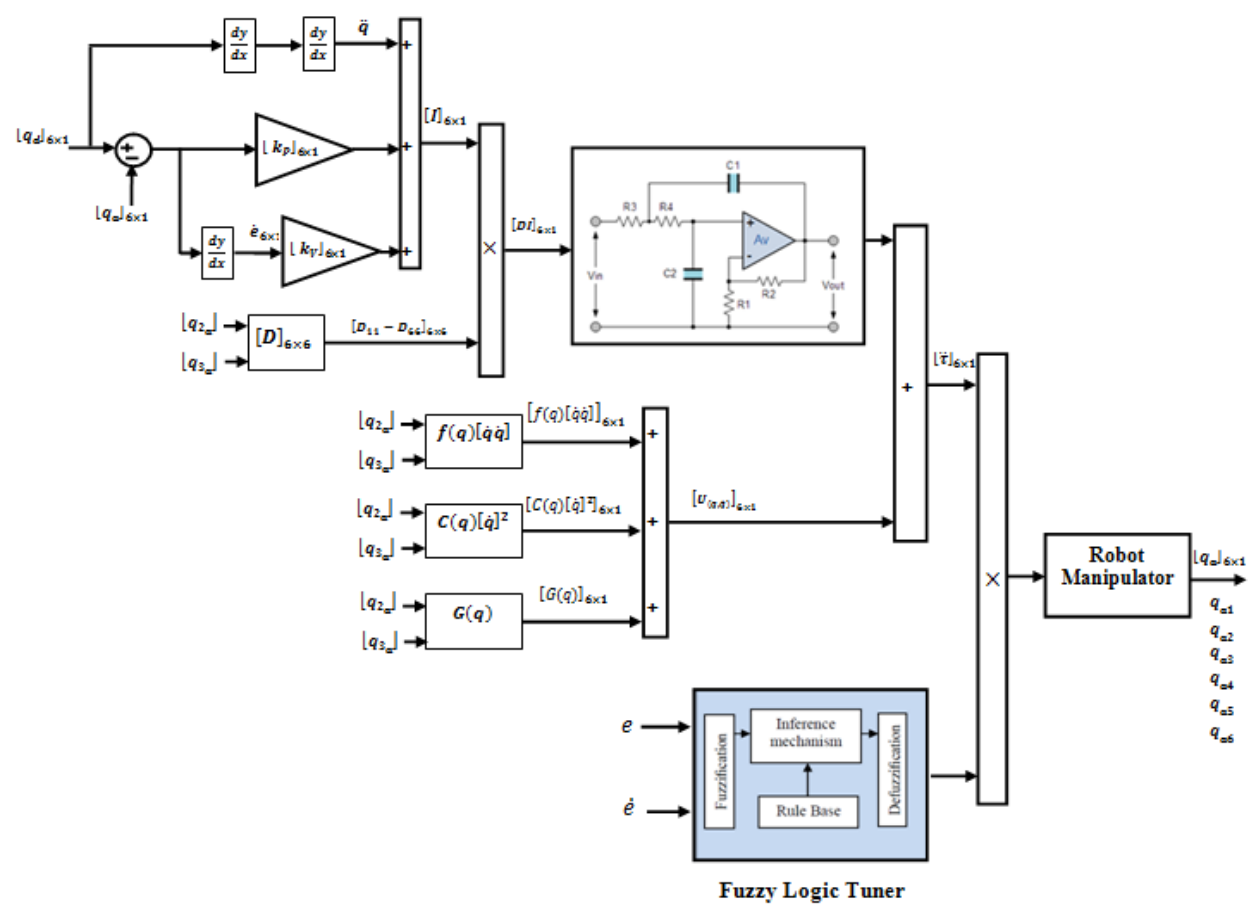

Figure 6. Block Diagram of LPF Fuzzy Computed Torque Controller 


\section{Results and Discussion}

LPF fuzzy computed torque controller was tested to Step response trajectory. In this simulation, to control position of continuum robot the first, second, and third joints are moved from home to final position without and with external disturbance. The simulation was implemented in MATLAB/SIMULINK environment. These systems are tested by band limited white noise with a predefined $40 \%$ of relative to the input signal amplitude. This type of noise is used to external disturbance in continuous and hybrid systems and applied to nonlinear dynamic of these controllers.

Tracking performances: Figure 7 shows the trajectory following in two type's methodology: LPF fuzzy CTC and conventional CTC. Due to the following graph, in certain conditions both methods have accepted performance. However CTC methodology has the better time response $\left(t_{r}=0.6 \mathrm{sec}\right)$ compare to LPF fuzzy CTC $\left(t_{r}=1 \mathrm{sec}\right)$ but conventional CTC has the steady state error $\left(e_{s s}=1 \times 10^{-3}\right.$ ) but in LPF fuzzy CTC steady state error near to zero. According to the following Figure both methods have about the same response in certain conditions.

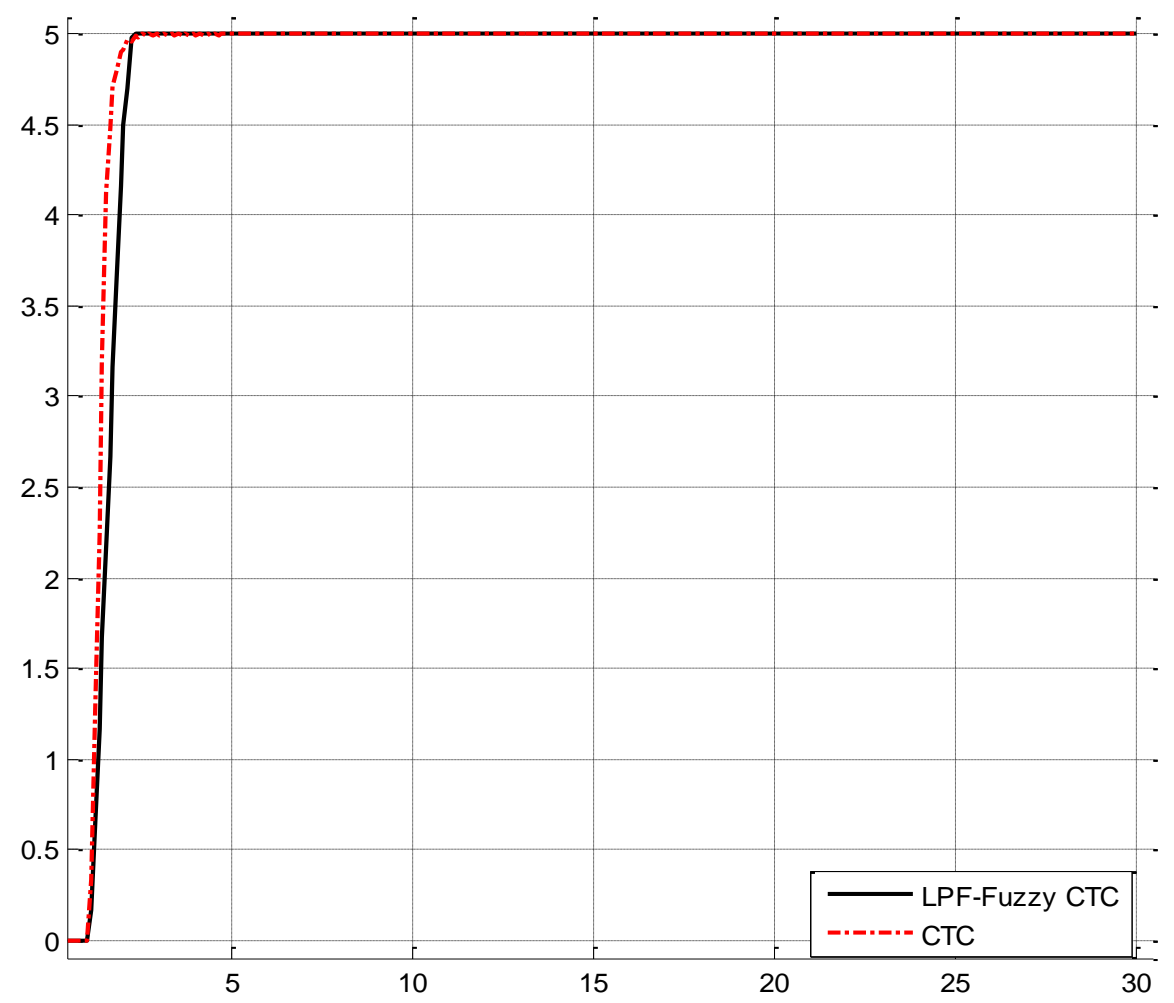

Fig 7: Trajectory follows: LPF Fuzzy CTC and CTC

Disturbance rejection: Figure 8 shows the power of high frequency oscillation in conventional CTC and LPF fuzzy CTC. According to the following graph, LPF fuzzy CTC reduce the high frequency noise which caused to instability to design controller. According to this method, in presence of $40 \%$ disturbance the rate of time response change in CTC is about $10 \%\left(t_{r}=0.7 \mathrm{sec}\right)$ but in LPF fuzzy CTC this rate is about $0 \%\left(t_{r}=1 \mathrm{sec}\right)$. The rate of overshoot in conventional CTC is $(O . S \%=6.4 \%)$ but the overshoot in LPF fuzzy CTC is 
$(O . S \%=0 \%)$. In point of steady state error views conventional CTC has high frequency oscillation and the steady state error is $\quad\left(e_{s s}=0.78\right)$ but in LPF fuzzy CTC is almost equal to zero.



Figure 8. Power of Disturbance Rejection: LPF Fuzzy CTC and CTC

\section{Conclusion}

In this research the application of communication in mechatronics is analysis. According to this paper, the low pass filter is design and applied to fuzzy computed torque controller. This method is used to reduce the high frequency oscillations which created by $40 \%$ external noise or hand tremors. Due to this method and based on result we can see that the rate of disturbance rejection which caused to robustness in LPF fuzzy CTC is about 100\%. It means that this method can eliminate the frequency oscillation which caused by external noise or hand tremors. The combination of these methods can make a new window industrial medical science.

\section{Acknowledgment}

The authors would like to thank the anonymous reviewers for their careful reading of this paper and for their helpful comments. This work was supported by the Iranian Institute of Advance Science and Technology Program of Iran under grant no. 2012-Persian Gulf-2A. 
Iranian center of Advance Science and Technology (IRAN SSP) is one of the independent research centers specializing in research and training across of Control and Automation, Electrical and Electronic Engineering, and Mechatronics \& Robotics in Iran. At IRAN SSP research center, we are united and energized by one mission to discover and develop innovative engineering methodology that solve the most important challenges in field of advance science and technology. The IRAN SSP Center is instead to fill a long standing void in applied engineering by linking the training a development function one side and policy research on the other. This center divided into two main units:

- $\quad$ Education unit

- $\quad$ Research and Development unit

\section{References}

[1] T. R. Kurfess, "Robotics and automation handbook: CRC", (2005).

[2] J. J. E. Slotine and W. Li, "Applied nonlinear control vol. 461: Prentice hall Englewood Cliffs", NJ, (1991).

[3] L. Cheng, "Multi-agent based adaptive consensus control for multiple manipulators with kinematic uncertainties", (2008), pp. 189-194.

[4] J. J. D'Azzo, "Linear control system analysis and design with MATLAB: CRC", (2003).

[5] B. Siciliano and O. Khatib, "Springer handbook of robotics: Springer-Verlag New York Inc.", (2008).

[6] I. Boiko, "Analysis of chattering in systems with second-order sliding modes", IEEE Transactions on Automatic Control, vol. 52, (2007), pp. 2085-2102.

[7] J. Wang, "Indirect adaptive fuzzy sliding mode control: Part I: fuzzy switching", Fuzzy Sets and Systems, vol. 122, (2001), pp. 21-30.

[8] F. Piltan, "Artificial Control of Nonlinear Second Order Systems Based on AFGSMC", Australian Journal of Basic and Applied Sciences, vol. 5, no. 6, (2011), pp. 509-522.

[9] V. Utkin, "Variable structure systems with sliding modes", Automatic Control, IEEE Transactions on, vol. 22, (2002), pp. 212-222.

[10] R. A. DeCarlo, "Variable structure control of nonlinear multivariable systems: a tutorial", Proceedings of the IEEE, vol. 76, (2002), pp. 212-232.

[11] K. D. Young, "A control engineer's guide to sliding mode control", (2002), pp. 1-14.

[12] S. Soltani and F. Piltan, "Design Artificial Nonlinear Controller Based on Computed Torque like Controller with Tunable Gain”, World Applied Science Journal (WASJ), vol. 14, no. 9, (2011), pp. 13061312.

[13] F. Piltan, S. Emamzadeh, S. Heidari, S. Zahmatkesh and K. Heidari, "Design Artificial Intelligent Parallel Feedback Linearization of PID Control with Application to Continuum Robot", International Journal of Engineering and Manufacturing, vol. 3, no. 2, (2013), pp. 51-72.DOI: 10.5815/ijem.2013.02.04

[14] A. Jahed, F. Piltan, H. Rezaie and B. Boroomand, "Design Computed Torque Controller with Parallel Fuzzy Inference System Compensator to Control of Robot Manipulator", International Journal of Information Engineering and Electronic Business, vol. 5, no. 3, (2013), pp. 66-77. DOI: 10.5815/ijieeb.2013.03.08

[15] M. Kazeminasab, F. Piltan, Z. Esmaeili, M. Mirshekaran and A. Salehi, "Design Parallel Fuzzy Partly Inverse Dynamic Method plus Gravity Control for Highly Nonlinear Continuum Robot", IJISA, vol. 6, no. 1, (2014), pp. 112-123. DOI: 10.5815/ijisa.2014.01.12.

\section{Authors}

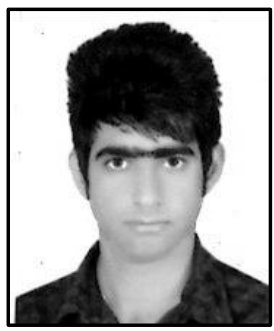

Mahdi Mirshekaran, he is currently working as a primary researcher in the laboratory of Control and Robotic, Institute of Advance Science and Technology, IRAN SSP research and development Center. His current research interests are in the area of nonlinear control, artificial control system and robotics. 


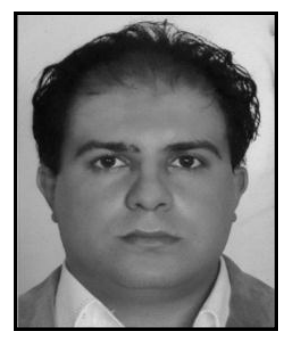

Farzin Piltan, he was born on 1975, Shiraz, Iran. In 2004 he is jointed Institute of Advance Science and Technology, Research and Development Center, IRAN SSP. Now he is a dean of Intelligent Control and Robotics Lab. He is led of team (47 researchers) to design and build of nonlinear control of industrial robot manipulator for experimental research and education and published about 54 Papers in this field since 2010 to 2012, team supervisor and leader (9 researchers) to design and implement intelligent tuning the rate of fuel ratio in internal combustion engine for experimental research and education and published about 17 Journal papers since 2011 to 2013, team leader and advisor (34 researchers) of filtering the hand tremors in flexible surgical robot for experimental research and education and published about 31 journal papers in this field since 2012 to date, led of team (21 researchers) to design high precision and fast dynamic controller for multi-degrees of freedom actuator for experimental research and education and published about 7 journal papers in this field since 2013 to date, led of team (22 researchers) to research of full digital control for nonlinear systems (e.g., Industrial Robot Manipulator, IC Engine, Continuum Robot, and Spherical Motor) for experimental research and education and published about 4 journal papers in this field since 2010 to date and finally led of team (more than 130 researchers) to implementation of Project Based-Learning project at IRAN SSP research center for experimental research and education, and published more than 110 journal papers since 2010 to date. In addition to 7 textbooks, Farzin Piltan is the main author of more than 115 scientific papers in refereed journals. He is editorial review board member for 'international journal of control and automation (IJCA), Australia, ISSN: 2005-4297; 'International Journal of Intelligent System and Applications (IJISA)', Hong Kong, ISSN:2074-9058; 'IAES international journal of robotics and automation, Malaysia, ISSN:2089-4856; 'International Journal of Reconfigurable and Embedded Systems', Malaysia, ISSN:2089-4864. His current research interests are nonlinear control, artificial control system and applied to FPGA, robotics and artificial nonlinear control and IC engine modeling and control.

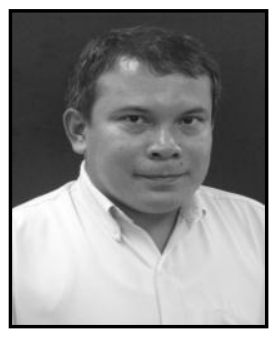

Nasri Sulaiman, he received his B. Eng from University of Putra Malaysia In 1994, M. Sc., From University Of Southampton, UK in1999, And Ph. D. degrees From University Of Edinburgh, Uk In 2007, respectively. He has more than 65 journal and conference papers. He is Currently A Senior Lecturer In The Department Of Electrical Engineering At University Putra Malaysia Of The Program For Signal Processing, And Evolvable Hardware (EHW) And Also Is Head Of Control And Automation Laboratory, Iranian Institute Of Advanced 
Science And Technology, Shiraz, Iran. He Is An Advisor In Spherical Motor Control.

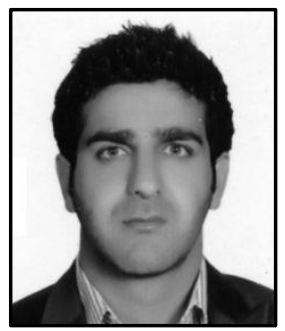

Alireza Salehi, he is currently working as a primary researcher in the laboratory of Control and Robotic, Institute of Advance Science and Technology, IRAN SSP research and development Center. His current research interests are in the area of nonlinear control, artificial control system and robotics.

Meysam Kazeminasab is currently working as a primary researcher in the laboratory of Control and Robotic, Institute of Advance Science and Technology, IRAN SSP research and development Center. His current research interests are in the area of nonlinear control, artificial control system and robotics.

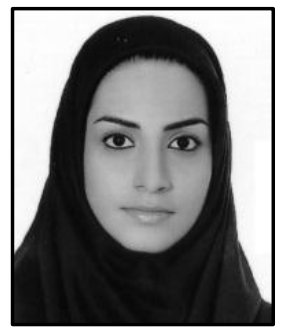

Zahra Esmaeili, she is currently working as a primary researcher in the laboratory of Control and Robotic, Institute of Advance Science and Technology, IRAN SSP research and development Center. Her current research interests are in the area of nonlinear control, artificial control system and robotics. 
International Journal of Bio-Science and Bio-Technology Vol.7, No.1 (2015) 